\title{
Deformation quantization and invariant distributions
}

\author{
Martin ANDLER a , Alexander DVORSKY ${ }^{\text {b }}$, Siddhartha SAHI ${ }^{\text {c }}$ \\ a Département de Mathematiques, Université de Versailles Saint Quentin, 78035 Versailles Cédex. \\ Courrier électronique : andler@math.uvsq.fr \\ b, c Mathematics Department, Rutgers University, New Brunswick, NJ 08903. \\ e-mail : dvorsky@math.rutgers.edu, sahi@math.rutgers.edu
}

\begin{abstract}
We study Kontsevich's deformation quantization for the dual of a finite dimensional Lie algebra $\mathfrak{g}$. Regarding elements of $\mathcal{S}(\mathfrak{g})$ as distributions on $\mathfrak{g}$, we show that the $\star$ multiplication operator $(r \mapsto r \star p)$ is a differential operator with analytic germ at 0 . We use this to establish a conjecture of Kashiwara and Vergne which, in turn, gives a new proof of Duflo's result on the local solvability of bi-invariant differential operators on a Lie group.
\end{abstract}

\section{Quantification par déformation et distributions invariantes}

Résumé. Nous étudions la quantification par déformations dans le cas du dual d'une algèbre de Lie de dimension finie $\mathfrak{g}$. Considérant les éléments de $\mathcal{S}(\mathfrak{g})$ comme des distributions de support 0 sur $\mathfrak{g}$, nous montrons que la $\star$-multiplication à droite $(r \mapsto r \star p)$ est un opérateur différentiel à germes analytiques en 0 . Nous utilisons ceci pour établir une conjecture de Kashiwara-Vergne, ce qui fournit une nouvelle démonstration du théorème de Duflo sur la résolubilité locale des opérateurs différentiels bi-invariants sur un groupe de Lie.

\section{Version française abrégée}

Soit $G$ un groupe de Lie réel connexe de dimension finie d'algèbre de Lie $\mathfrak{g}$, et soit $\mathcal{U}=\mathcal{U}(\mathfrak{g})$ et $\mathcal{S}=\mathcal{S}(\mathfrak{g})$, respectivement l'algèbre enveloppante et l'algèbre symétrique de $\mathfrak{g}$, que nous identifions à l'algèbre de convolution des distributions de support 1 sur le groupe $G$, et à l'algèbre de convolution des distributions de support 0 sur $\mathfrak{g}$. Notons $\mathcal{I}=\mathcal{S}^{\mathfrak{g}}, \mathcal{Z}=\mathcal{U}^{\mathfrak{g}}$ les sous-algèbres d'invariants correspondantes.

Soit $\mathbf{U}$ et $\mathbf{S}$ les espaces de germes en 1 et 0 de distributions sur $G$ et $\mathfrak{g}$, et $\mathbf{Z}$ et $\mathbf{I}$ les sous-espaces d'invariants correspondants. Nous avons les diagrammes commutatifs

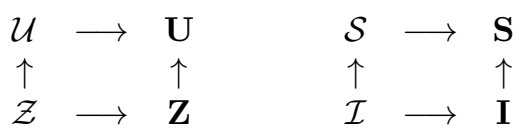

Soit $\exp _{*}: \mathbf{S} \rightarrow \mathbf{U}$ et $\exp ^{*}: \mathbf{U} \rightarrow \mathbf{S}$ l'image directe et inverse au sens des (germes de) distributions. Soit $q$ la fonction analytique sur un voisinage de 0 dans $\mathfrak{g}$ :

$$
q(x):=\sqrt{\operatorname{det}\left(\frac{e^{\operatorname{ad}(x) / 2}-e^{-\operatorname{ad}(x) / 2}}{\operatorname{ad}(x)}\right)} .
$$

Soit $\eta$ l'application de $S$ dans $U$

$$
\eta(p)=\exp _{*}(p q)
$$




\section{Andler, A. Dvorsky, S. Sahi}

Utilisant la méthode des orbites de Kirillov, Duflo [Du] a montré que la restriction de $\eta$ à $\mathcal{I}$ est un isomorphisme d'algèbres de $\mathcal{I}$ sur $\mathcal{Z}$, c'est-à-dire :

$$
\eta\left(p_{1} *_{\mathfrak{g}} p_{2}\right)=\eta\left(p_{1}\right) *_{G} \eta\left(p_{2}\right) \quad \text { pour } p_{1}, p_{2} \in \mathcal{I},
$$

où $*_{G}$ et $*_{\mathfrak{g}}$ sont respectivement le produit de convolution sur le groupe $G$ et l'algèbre de Lie $\mathfrak{g}$.

Soit $\mathfrak{D}$ l'algèbre des germes en 0 d'opérateurs différentiels à coefficients analytiques sur $\mathfrak{g}$. Soit $\mathfrak{R}$ l'idéal à droite de $\mathfrak{D}$ engendré par les germes de champs de vecteurs $\operatorname{adj}_{a}$ définis par l'action adjointe de $G$ sur $\mathfrak{g}$ :

$$
\operatorname{adj}_{a}(f)(x)=\left.\frac{d}{d t} f(\exp (-t a) \cdot x)\right|_{t=0}, a \in \mathfrak{g} .
$$

Naturellement, $\mathbf{S}$ est un $\mathfrak{D}$-module à droite - nous noterons donc l'action de $\mathfrak{D}$ sur les distribution à droite.

Nous démontrons le résultat suivant, conjecturé en général, et démontré dans le cas résoluble, par Kashiwara -Vergne [KV, Corollary 1].

Theorème 1. - Pour $p$ dans $\mathcal{I}$, l'opérateur $D_{p}: \mathbf{S} \rightarrow \mathbf{S}$ appartient à $\mathfrak{R}:$

$$
P \cdot D_{p}=\exp ^{*}\left(\eta\left(P *_{\mathfrak{g}} p\right)-\eta(P) *_{G} \eta(p)\right) .
$$

Comme cela est expliqué dans $[\mathrm{KV}]$, ce résultat a plusieurs conséquence importantes. D'abord, comme les éléments de $\mathfrak{R}$ annulent les distributions dans I nous obtenons :

Theorème 2. - Pour tout $p$ dans $\mathcal{I}$ et $P$ dans $\mathbf{I}$, nous avons

$$
\eta\left(P *_{\mathfrak{g}} p\right)=\eta(P) *_{G} \eta(p) .
$$

Rappelons ensuite qu'une distribution $Q$ sur $G$ est appelée distribution propre s'il existe un caractère $\chi: \mathcal{Z} \longrightarrow \mathbf{C}$ tel que

$$
Q *_{G} z=\chi(z) Q \text { pour tout } z \text { dans } \mathcal{Z} .
$$

On définit de même les distributions propres sur l'algèbre de Lie; nous obtenons le

TheORÈme 3. - L'application $\eta$ envoie les germes de distributions propres et invariantes sur l'algèbre de Lie sur ceux du groupe.

Ces résultats ont des conséquences importantes pour l'analyse des opérateurs différentiels biinvariants sur $G$ (voir version en anglais ci-dessous).

Nous démontrons le Théorème 1 en nous appuyant sur le travail remarquable de M. Kontsevich sur la formalité du complexe de Hochschild pour une variété différentiable [Ko]. Les étapes de la démonstration sont les suivantes. On démontre d'abord que, dans notre cas, on peut poser $h=1$ dans le produit $\star$, puis que, pour $p$ fixé dans $\mathcal{S}(\mathfrak{g})$, l'application $\partial_{p}^{\star}: r \in \mathcal{S} \mapsto r \star p$ appartient à $\mathfrak{D}$. Nous démontrons ensuite qu'une certaine série formelle considérée par Kontsevich [Ko] converge dans un voisinage de 0 dans $\mathfrak{g}$ vers une fonction $\tau(x)$. On démontre ensuite que pour $p \in \mathcal{I}$ l'opérateur $T_{p}=\partial_{p} \tau-\tau \partial_{p \tau}^{\star}$ appartient à $\mathfrak{R}$, où $\partial_{p}$ est l'opérateur de convolution par $p$ dans $\mathfrak{g}$. On compare enfin $T_{p}$ et $D_{p}$ en démontrant la formule $D_{p}=T_{p} \tau^{-1} q$. 


\section{Invariant distributions}

Let $G$ be a finite dimensional real Lie group with Lie algebra $\mathfrak{g}$. We write $\mathcal{U}=\mathcal{U}(\mathfrak{g}), \mathcal{S}=\mathcal{S}(\mathfrak{g})$ for the enveloping and symmetric algebras of $\mathfrak{g}$, and denote by $\mathcal{I}=\mathcal{S}^{\mathfrak{g}}, \mathcal{Z}=\mathcal{U}^{\mathfrak{g}}$ the subalgebras of $\mathfrak{g}$-invariants.

Let $\mathbf{U}$ and $\mathbf{S}$ be the spaces of germs at 1 and 0 of distributions on $G$ and $\mathfrak{g}$, and let $\mathbf{Z}$ and $\mathbf{I}$ be the subspaces of $\mathfrak{g}$-invariant distributions. We identify $\mathcal{U}$ and $\mathcal{S}$ with the convolution algebras of distributions with point support $1 \in G$ and $0 \in \mathfrak{g}$. Then we have the following inclusions

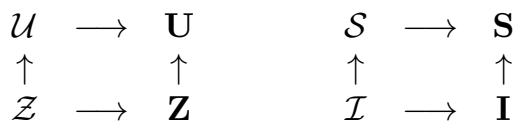

We denote by $\exp _{*}: \mathbf{S} \longrightarrow \mathbf{U}$ and $\exp ^{*}: \mathbf{U} \longrightarrow \mathbf{S}$ the pushforward and pullback of distributions (germs) under the exponential map; and define $\eta: \mathbf{S} \longrightarrow \mathbf{U}$ by

$$
\eta(p)=\exp _{*}(p q)
$$

where $q$ is the following analytic function on $\mathfrak{g}$ :

$$
q(x):=\sqrt{\operatorname{det}\left(\frac{e^{\operatorname{ad}(x) / 2}-e^{-\operatorname{ad}(x) / 2}}{\operatorname{ad}(x)}\right)} .
$$

Using the orbit method initiated by Kirillov, Duflo [Du] proved that the restriction of $\eta$ to $\mathcal{I}$ is an algebra isomorphism from $\mathcal{I}$ to $\mathcal{Z}$, i.e.

$$
\eta\left(p_{1} *_{\mathfrak{g}} p_{2}\right)=\eta\left(p_{1}\right) *_{G} \eta\left(p_{2}\right) \quad \text { for } p_{1}, p_{2} \in \mathcal{I}
$$

where $*_{\mathfrak{g}}$ and $*_{G}$ denote convolution in the Lie algebra and Lie group respectively.

Let $\mathfrak{D}$ be the algebra of germs at 0 of analytic coefficient differential operators on $\mathfrak{g}$, and let $\mathfrak{R}$ be the right ideal of $\mathfrak{D}$ generated by the germs of the adjoint vector fields

$$
\operatorname{adj}_{a}(f)(x)=\left.\frac{d}{d t} f(\exp (-t a) \cdot x)\right|_{t=0}, a \in \mathfrak{g} .
$$

By virtue of the pairing between functions and distributions, $\mathbf{S}$ is naturally a right $\mathfrak{D}$-module, and to emphasise this fact we shall write the action on distributions on the right.

We shall establish the following result which was conjectured by Kashiwara and Vergne, and proved by them for solvable groups [KV, Corollary 1].

Theorem 1. - For $p$ in $\mathcal{I}$, the following differential operator $D_{p}: \mathbf{S} \rightarrow \mathbf{S}$ lies in $\mathfrak{R}$ :

$$
P \cdot D_{p}=\exp ^{*}\left(\eta\left(P *_{\mathfrak{g}} p\right)-\eta(P) *_{G} \eta(p)\right) .
$$

As is explained in $[\mathrm{KV}]$, this result has several important consequences. First, since operators from $\mathfrak{R}$ annihilate distributions from $\mathbf{I}$, we obtain the following extension of the Duflo isomorphism :

Theorem 2. - For $p$ in $\mathcal{I}$ and $P$ in $\mathbf{I}$, we have

$$
\eta\left(P *_{\mathfrak{g}} p\right)=\eta(P) *_{G} \eta(p) .
$$


Next, recall that a distribution $Q$ on $G$ is called an eigendistribution if there is a character $\chi: \mathcal{Z} \longrightarrow \mathbf{C}$ such that

$$
Q *_{G} z=\chi(z) Q \text { for all } z \text { in } \mathcal{Z}
$$

Eigendistributions on the Lie algebra are defined similarly, and we obtain the following:

THEOREM 3. - The map $\eta$ takes germs of invariant eigendistributions on the Lie algebra to those on the Lie group.

Another consequence is the following important result, which was conjectured by L. Schwartz and proved by Duflo in $[\mathrm{Du}]$ by different methods. (See also $[\mathrm{He}],[\mathrm{Ra}],[\mathrm{DR}]$ for various special cases):

COROLlary. - Every nonzero bi-invariant differential operator on $G$ is locally solvable.

This follows immediately from Theorem 2 and Rais' lemma from [Ra] on the existence of invariant fundamental solutions on the Lie algebra $\mathfrak{g}$.

\section{Deformation quantization}

We will deduce Theorem 1 from careful analysis of M. Kontsevich's remarkable result on the formality of the Hochschild complex of a smooth manifold [Ko].

Kontsevich's main result leads to the construction of an explicit $\star$-product on the algebra of functions on an arbitrary smooth Poisson manifold $(X, \gamma)$. This $\star$-product is given by a formal power series of bidifferential operators (depending on a parameter $\hbar$ ).

In the special situation when $X=\mathfrak{g}^{*}$ is the dual of a finite dimensional Lie algebra $\mathfrak{g}$, and $\gamma$ is the usual Poisson bracket (dual to the Lie bracket); we can set $\hbar=1$ and Kontsevich's $\star$-product descends to an actual product on the algebra of polynomial functions on $\mathfrak{g}^{*}$, which can be naturally identified with $\mathcal{S}=\mathcal{S}(\mathfrak{g})$.

If, as before, we consider $\mathcal{S}$ as the algebra of distributions with point support (at $0 \in \mathfrak{g}$ ), then the $\star$-product can be viewed as a new convolution on $\mathcal{S}$. The advantage of the distributional point of view is the following key result:

Theorem 4. - Given $p \in \mathcal{S}$ there is a unique element $\partial_{p}^{\star}$ in $\mathfrak{D}$ such that for all $r \in \mathcal{S}$

$$
r \star p=r \cdot \partial_{p}^{\star} .
$$

(As explained in the previous section, we are writing differential operators on the right.)

The proof of this result involves examining the Kontsevich $\star$-product formula, replacing multiplications by differentiation and vice versa. This results in an expression for $\partial_{p}^{\star}$ as an infinite sum of polynomial coefficient differential operators of bounded order. The coefficients of the sum are given by certain integrals which, while hard to compute, can be readily estimated to deduce Theorem 4. The linearity of $\gamma$ is crucial in these considerations.

Next, in [Ko, 8.3.3] Kontsevich introduces a certain formal power series

$$
S_{1}(x)=\exp \left(\sum_{k=1}^{\infty} c_{2 k} \operatorname{tr}(\operatorname{ad} x)^{2 k}\right),
$$

where the constants $c_{2 k}$ (denoted $c_{2 k}^{(1)}$ in [Ko]) are expressed as integrals of certain smooth differential forms ("angle forms") over certain compact manifolds with corners (compactified configuration spaces, introduced in $[\mathrm{FM}])$. It can be checked that

$$
c_{n} \sim \alpha^{n}
$$


for some positive constant $\alpha$. Hence in a neighborhood of 0 the power series $S_{1}(x)$ converges to an analytic function which we shall denote by $\tau(x)$.

TheOREm 5. - For $p \in \mathcal{I}$, the operator $T_{p}=\partial_{p} \tau-\tau \partial_{p \tau}^{\star}$ belongs to $\mathfrak{R}$.

Here $\partial_{p \tau}^{\star}$ is the differential operator defined in Theorem 4 and $r \cdot \partial_{p}:=r *_{\mathfrak{g}} p$.

To establish this result, we study the expression

$$
\left(r *_{\mathfrak{g}} p\right) \tau-(r \tau) \star(p \tau)
$$

for $r \in \mathcal{S}$ and $p \in \mathcal{I}$, as in section 8.2 of [Ko]. This enables us to express $T_{p}$ as an infinite sum of differential operators each belonging to $\mathfrak{R}$, and Theorem 5 follows from Theorem 4.

Theorem 1 is now a consequence of the following lemma:

Lemma 1. - For $p \in \mathcal{S}$, we have

$$
D_{p}=T_{p} \tau^{-1} q
$$

To prove this lemma it is enough to show that $r \cdot D_{p}=r \cdot\left(T_{p} \tau^{-1} q\right)$ for $r$ in $\mathcal{S}$. In turn, this follows from the fact that there is a canonical isomorphism $\kappa: \mathcal{S} \rightarrow \mathcal{U}$ (denoted $I_{\text {alg }}^{-1}$ in [Ko]) which satisfies the relations $[\mathrm{Ko}, 8.3 .1]$

$$
\kappa(r \star p)=\kappa(r) *_{G} \kappa(p)
$$

and $[\mathrm{Ko}, 8.3 .4]$

$$
\kappa(r \tau)=\eta(r)=\exp _{*}(r q) \text { for } r \in \mathcal{S} .
$$

Acknowledegment. - The authors would like to thank M. Kontsevich for sharing his insights with us, especially for explaining the proof of Theorem 8.2 of [Ko]. Moreover, this paper would not have been written without the help of I. Gelfand and F. Knop, and that of the other participants of the Gelfand seminar at Rutgers, especially R. Wilson, C. Weibel, J. Barros-Neto, M. Nathanson, J. Lagarias, D. Stone, O. Stoyanov, and A. Kazarnovski-Krol, who helped us tremendously in the enjoyable task of discussing, dissecting, and digesting Kontsevich's formidable manuscript.

\section{Bibliographic References}

[Du] M. Duflo, Opérateurs différentiels bi-invariants sur un groupe de Lie, Ann. Sc. Éc. Norm. Sup 10 (1977), 267-288

[DR] M. Duflo, M. Rais, Sur l'analyse harmonique sur les groupes de Lie résolubles, Ann. Sc. Éc. Norm. Sup 9 (1976), 107-114.

[FM] W. Fulton, R. MacPherson, Compactification of configuration spaces, Ann. of Math. 139 (1994), 183-225.

[He] S. Helgason, The surjectivity of invariant differential operators on a symmetric space, Ann. of Math. 98 (1973), 451-479.

[KV] M. Kashiwara, M. Vergne, The Campbell-Hausdorff formula and invariant hyperfunctions, Inventiones Math. 47 (1978), 249-272.

[Ko] M. Kontsevich, Deformation quantization of Poisson manifolds I, e-print math.QA/9709040).

[Ra] M. Rais, Solutions élémentaires des opérateurs différentiels bi-invariants sur un groupe de Lie nilpotent, C. R. Acad. Sci. 273 (1971), 495-498. 